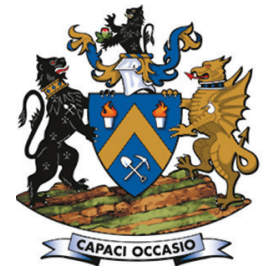

\title{
Application of a content management system for developing equipment safety training courses in surface mining
}

\author{
L. Zujovic ${ }^{1}$, V. Kecojevic ${ }^{1}$, and D. Bogunovic ${ }^{2}$
}

\begin{abstract}
Affiliation:
1 Department of Mining

Engineering, West Virginia

University, Morgantown, WV,

USA.

2 North American Coal

Corporation, Farmington, NM USA.
\end{abstract}

Correspondence to:

L. Zujovic

Email:

Iz0011@mix.wvu.edu

Dates:

Received: 30 May 2020

Revised: 13 Aug. 2020

Accepted: 13 Aug. 2020

Published: August 2020

\section{How to cite:}

Zujovic, L., Kecojevic, V., and

Bogunovic, D.

Application of a content

management system for

developing equipment safety

training courses in surface

mining.

The Southern African Institute of

Mining and Metallurgy

DOI ID:

http://dx.doi.org/10.17159/2411-

9717/1233/2020

ORCID ID:

L. Zujovic

https://orchid.org/0000-0002-

6197-1041

\section{Synopsis \\ Web-based training (WBT) has become a widely popular training option that can be applied throughout a variety of areas and industries. Mining is a challenging industry that is continuously searching for improvements in its safety training processes. We used the content management system (CMS) WordPress to develop a web-based application to support traditional training in the mining industry. The study focuses on introducing operators of heavy machinery in surface mining operations to pre- shift machine inspections. WordPress does not require technical skills, and in addition to its default features, it offers a vast number of useful plugins. This project implements several plugins to create an application with training courses. In this paper we present both the process of creating a training course and an example of a developed course. Such training applications can be used on the internet or through a local area network.}

\section{Keywords}

web-based training, WordPress, safety training, surface mining.

\section{Introduction}

Sustaining the quality of equipment safety training and education is one of the major responsibilities for any mining company, including both management and workforce. Heavy machinery, such as haul-trucks, dozers, and excavators, is essential for every mine operation and project. In the perennial challenge to secure safe working environments, it is of paramount importance for mining professionals to establish safety training. Additionally, training is mandated by the Mine Safety and Health Administration (MSHA, 30 CFR, Parts 46 and 48). Such training is given not only to miners assigned for duties for which they do not have previous experience (e.g. mobile equipment operators), but also to experienced miners who must undergo routine refresher training.

Kral (2002) stated that one of the best ways to retain personnel at mines is by promoting a safe work environment and training them to operate equipment properly. Peters, Vaught, and Mallet (2010) discussed thirty years of NIOSH (National Institute for Occupational Safety and Health) and US Bureau of Mines research on health and safety training in mining and made suggestions for major future improvements in miners' safety training. Gao et al. (2019) investigated the role of safety management in the development of safety culture. Out of the four safety management practices that they found to have a positive impact on safety culture, one was related to safety training, and another to inspection and monitoring. Brnich et al. (2002) suggested training improvements in the form of short, five-toseven minute videotapes about fire prevention in the context of the miners' workplace. They concluded that these videos had improved employee awareness about hazards and that such modules can be expanded to other topics.

Researchers have discussed different approaches to training and education in mining processes. Akkoyun and Careddu (2013) developed a computer program for teaching and learning in mining engineering-related courses. The authors selected an open pit magnesite mine to create a simulation application, concluding that such computer applications can be used for lectures in surface mining, drilling, blasting, mining economy, etc. Zhang and Kecojevic (2016) investigated both fundamental and traditional approaches as well as innovative and creative ones to mitigate the causes of incidents at mining sites. These authors also suggested improvements to training and education systems.

Despite being a relatively new technology, web-based training (WBT) and internet education are now widely used and supported. Many platforms such as Udemy and Coursera offer a wide range of courses in computer science, business, leadership, and many other disciplines. At the beginning of 


\section{Application of a content management system for developing equipment safety training courses}

this century, Fahy (2004) addressed the potential uses of WBT, suggesting that such delivery models would continue to grow as technologies become more available. He concludes that the potential strength of WBT might improve many kinds of training. Chamers and Lee (2004) reviewed WBT in the late 1990s by studying examples from several American corporations. Chan and Ngai (2007) discussed the implementation of WBT in ten different organizations in Hong Kong, one of which was in business with heavy machinery.

In the past two decades, the content management system (CMS) concept has evolved due to the importance of managing and maintaining data available on the internet (Fernandes and Vidyasagar, 2015a). Therefore, it is important not only to develop a web application, but also to consistently manage and manipulate its content. A CMS represents a computer application that enables content by using a central interface; this allows one to build an online computer program that is easier to use and offers more functionality than a regular website. For example, content management systems can be employed for user and media management.

As outlined by Manoj and Asoke (2016a), there are two primary components of a CMS: a content management application (CMA) and a content delivery application (CDA). The CMA assists in creating, modifying, and deleting content. As such, a person who is managing the content (as an administrator) can create new content and organize existing content, even without prior programming skills. The CDA updates the website and allows its content to be utilized. Some of the advantages of the CMS include time-saving in terms of developing the content, the possibility to create strong passwords for security reasons, and the use of themes and plugins that can support the process of creating or changing the CMS.

Figure 1 oulines the web content management system (Manoj and Asoke, 2016b). The person who is creating and organizing content utilizes coding skills, manipulates the database (by inserting new material such as images, videos, sound files, and text), and uses different plugins and templates to create the website. Therefore, the application, database, and server are establishing the back-end capabilities. Such an application can then be presented in a front-end environment. Here, the term 'front end' alludes to the user interface; the visitors can directly interact with the website only to see the data, objects, and other content inserted by the content organizer. Even though the front end and back end serve different purposes in the process of creating web applications, both should be user-friendly and easily accessible. After set-up, the CMS generally does not require specialized technical skills. The content manager can create, sustain, and organize the website without having coding knowledge. Such knowledge is, of course, helpful for improving the quality of the website, but not essential.

Today, with the rapid expansion of digital data and computer technology, a number of open-source CMA applications are publicly available and can be used to create and publish web-based software. CMS itself offers a practical solution for developing various types of websites and organizing their content. For example, blogging sites, portals, e-commerce sites, and enterprise websites can be developed within a CMS. Some of the currently popular CMS platforms include WordPress, Drupal, Magento, OpenChart, Wix, and Joomla.

The main objective of this study was to show the potential of using the free and open-source CMS WordPress to create safety training courses in the mining industry. The specific research objectives were to:

(i) Design and develop a web-based application for safety training purposes in the surface mining industry, emphasizing introduction to heavy machinery through pre-shift machine inspections

(ii) Determine main and supplementary plugins useful for the application, then utilize them to create an application with different virtual tours and quizzes

(iii) Describe the process of creating a training course through the developed template and give examples of developed courses.

\section{Methodology}

We used a popular CMS called WordPress to develop the webbased training application. This CMS was created in 2003 as a blogging platform and became popular due to its user-friendly interface (WordPress, 2020). This paper shows the procedure by which the new training course was developed, what plugins were used, and how the developed application appears in a digital environment.

Three experienced operators tested the developed application and reported universally positive feedback. The average age of the operators was fifty years and their average experience was twenty years.

\section{The content management system (CMS)}

The most notable feature of WordPress is that no programming experience is needed to work within this platform. WordPress offers thousands of open-source plugins that can be utilized in the process of developing a given application. By incorporating this vast variety of plugins, WordPress offers tremendous potential for building a more efficient web-based program for the end-user. Plugins allow users to create content with ease, insert new content, modify the application, and to improve the application not only on PCs but also through mobile devices. Many authors have discussed and implemented WordPress in their research. Patel, Rathod, and Parikh (2011) analysed and compared three different CMS applications - WordPress, Joomla, and Drupal. In their statistical analysis, they stated that WordPress is the most suitable for live websites. Ghorecha and Bhatt (2013) investigated the same three applications and concluded that while each has some positive features, WordPress is most useful for its plugins.

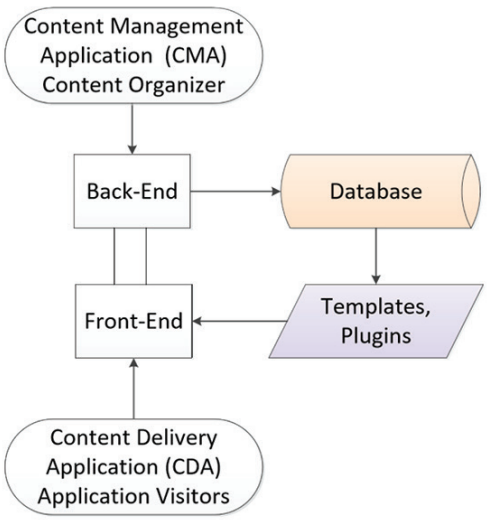

Figure 1-Web content management system 


\section{Application of a content management system for developing equipment safety training courses}

Researchers have also utilized WordPress for different purposes. Namestovski, Takacs, and Arsovic (2012) presented the uses of WordPress and Moodle for e-learning. They stated that the large number of plugins, templates, and the flexible use of media are clear advantages of WordPress. They concluded that both platforms provide good support to traditional education. Avila et al. (2016) used WordPress to develop an ePortfolio environment to support learning in medical education. The authors found that it is possible to build a system that can be used for different activities, such as storing multimedia, web publishing, studying, etc. Fernandez and Vidyasagar (2015b) analysed the use of WordPress in the area of digital marketing, discussing WordPress plugins and how they can extend the use of WordPress to perform almost any task. Baines (2015) analysed the book WordPress for Libraries and stated that WordPress can be useful for organizing, searching, and accessing information. The author concludes that the use of WordPress will continue to expand. This variety of research conducted in different areas shows the potential of using WordPress for various purposes. Cabot (2018) stated that the research community has much to contribute to the future of WordPress and that there should be more research on the WordPress platform. In addition, different programming languages can be used to provide support in creating the application.

\section{Development}

There were three major phases in building the web-based training application that would create and present training courses for heavy machine operators. Figure 2 shows the steps in creating the application with WordPress.

The first phase was to install WordPress and set up the server with the database. At first a virtual server with database was used; then the application was placed on a live server.

The second phase was designing the template of the application. First, the page builder was created and used to customize individual web pages. The page builder consists of three menu options - Courses, Instructions, and Contents. The options, which are not a default part of WordPress CMS, were specially developed for this project. These were inserted manually to create web pages in the application.

Training manuals for different machines were handed out while designing the application. These contained information about the particular machines located at the mine site and included explanations and guidelines for the training of heavy machine operators. In addition to the training manuals, 360-degree images, videos, and standard 2D images were gathered to further supplement the training. Other template options - main and supplementary plugins - were also implemented within the developed web pages.

Phase three, the final menu, is the result of phases one and two and presents a dashboard which consists of nine menu options (Figure 2). All of these nine options present in the template are used in the process of creating a new training course for the chosen mining machines.

Training materials were then incorporated into a media library within the developed web application. These were used through the main plugins and the page builder in order to create different training courses.

At this point, the researchers had yet to decide what plugins would best fit this project. As stated earlier, WordPress offers a large number of plugins that can be installed and used for different purposes in the web-based application. Among these, we identified and selected the plugins that would be suitable as tools for the development of the new application.

It was important to determine which WordPress plugins allowed for the use of 360-degree materials (images and videos). The advantages of the plugins and their simplicity of usage were considered. Thus, three appropriate plugins were selected for creating the training courses, two of which support 360-degree materials. The selected plugins are:

> iPanorama 360 (iPanorama 360, 2019)

> Wpvr (WordPress, 2019)

- Quizzes/Surveys Master (Quiz Master, 2019).

Apart from these three plugins, a few supplementary plugins were added to improve user experience. Those plugins were'Catch IDs', 'Duplicate Post', and 'Regenerate Thumbnails'. These additional plugins are not necessary to the functionality of the application; however, they improve the general user experience and user performance. A brief explanation of the main and supplementary plugins and the three default menu options that are utilized in the application is given in Figure 3.

\section{Results and discussion}

The training template, developed as the main feature of the webbased training, allowed for the creation of a new training course for a specific machine. Before a new course can be created, any relevant media files must be first inserted into the program. This can be done by using the 'Media' option from the menu. Users can either browse to add a new media file (a) or drag and drop a file (b), as indicated in Figure 4. After that, several steps (Figure 5) are followed in the process of creating a new course. The labels

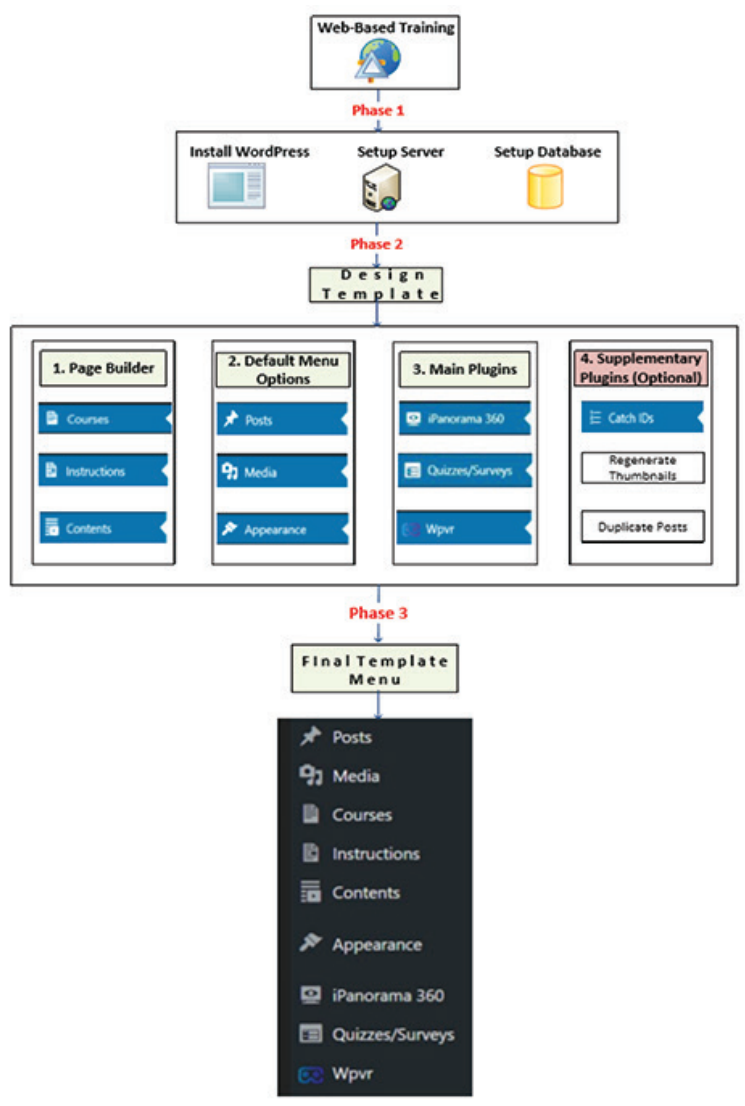

Figure 2-Creating web-based application 


\section{Application of a content management system for developing equipment safety training courses}

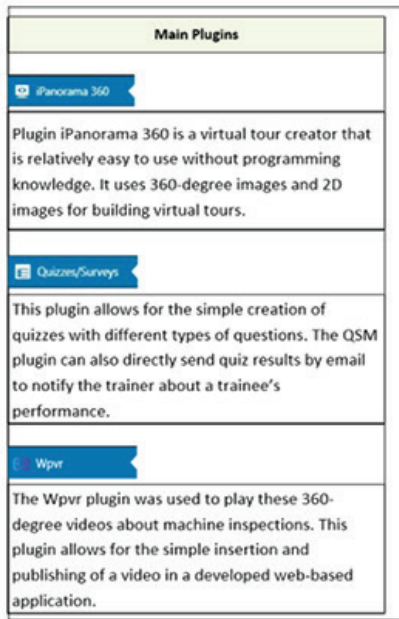
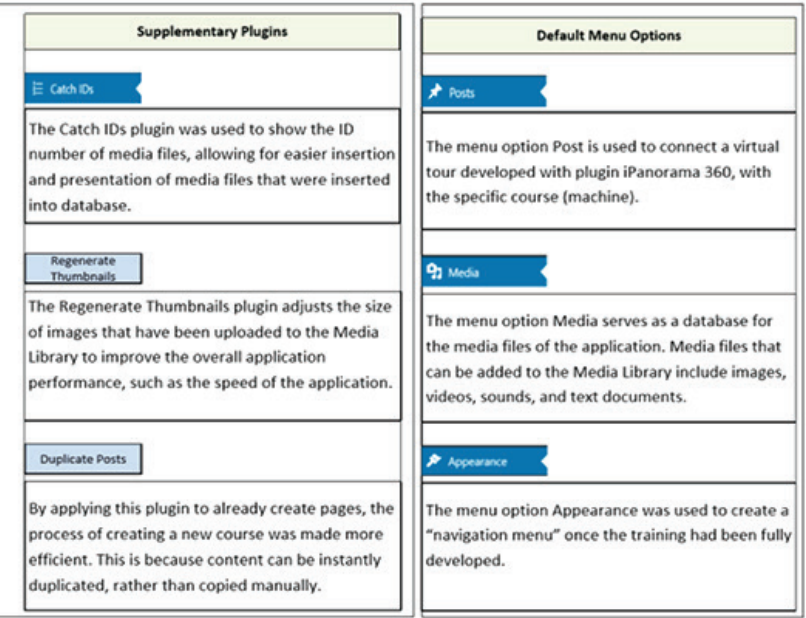

Figure 3-Description of plugins and default menu options

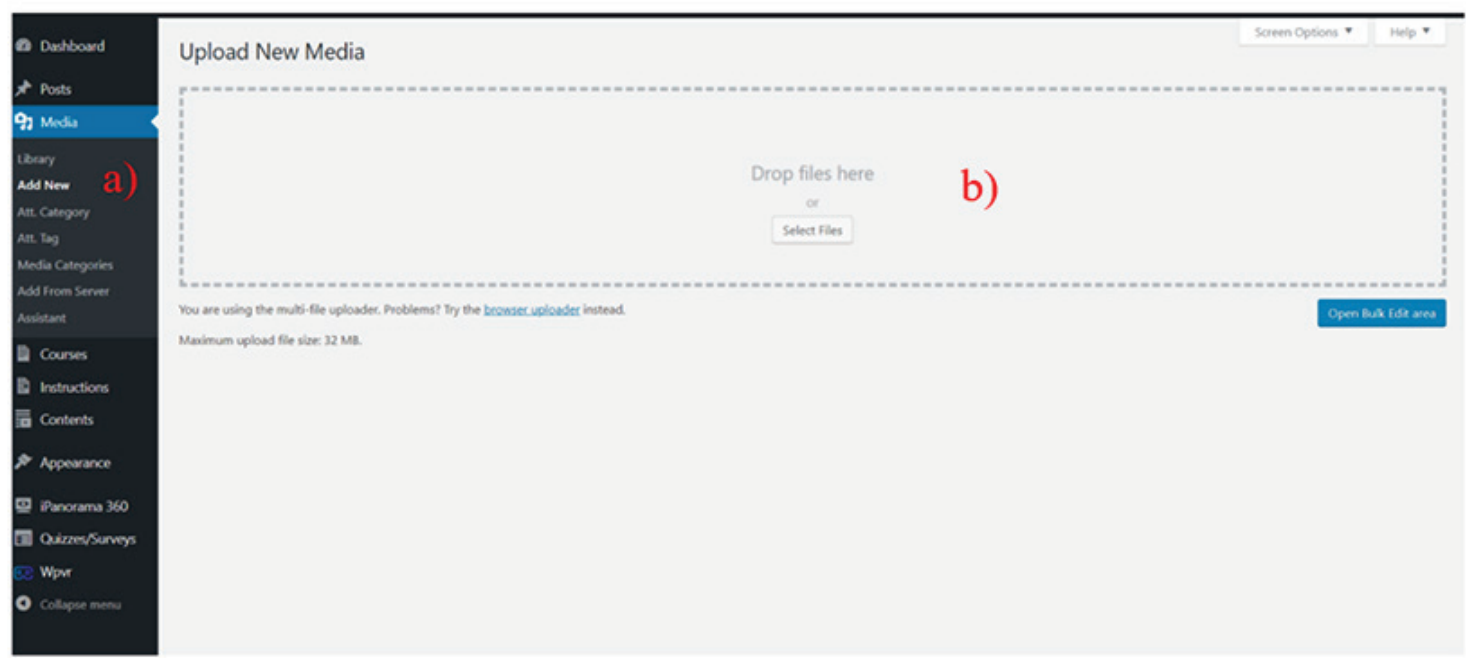

Figure 4-Insert media

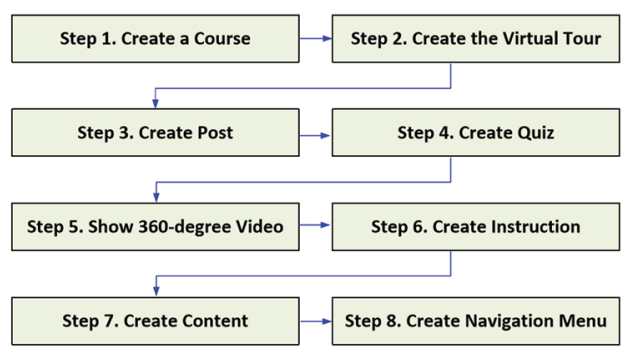

Figure 5-Process of creating a new training course

'a', 'b', and so on in the following figures are either red or black and are used to mark the relevant options. The colour scheme changes to enhance visualizations by taking the background colour into consideration.

Each menu option has a fillable form in which users can enter text and insert training materials such as MS Word or PDF documents, images, videos, etc. Users utilize the main plugins to create virtual tours and quizzes. Every virtual tour and quiz should be connected with its associated course.
The first step in the process of creating a new training course (Figure 6) is by accessing the 'Courses' menu option. Here, a course creator should first enter the course title (a) and the course name (b). The creator should then insert the course image (c), which is a 2D photo of a machine from the media library. Finally, a mine location (d) should be selected, and the new course can then be published (e).

The second step employs the plugin 'iPanorama 360' to create virtual tours for the machine inspections. This can be done by combining 360-degree images and 2D images (iPanorama 360, 2019).

In the third step, after the user has created a virtual tour, it is necessary to create a post for the same tour. The menu option 'Post' (Figure 7) can be used to connect a virtual tour with a specific course (machine) so that the virtual tour can appear within the course. The title of the new post should now be added (a). In the label (b), a shortcode of the created virtual tour should be inserted. Every virtual tour has a unique shortcode that is generated automatically after the tour has been created. The shortcode is given in the following format: [iPanorama $\mathrm{id}=" 000 "]$. 


\section{Application of a content management system for developing equipment safety training courses}

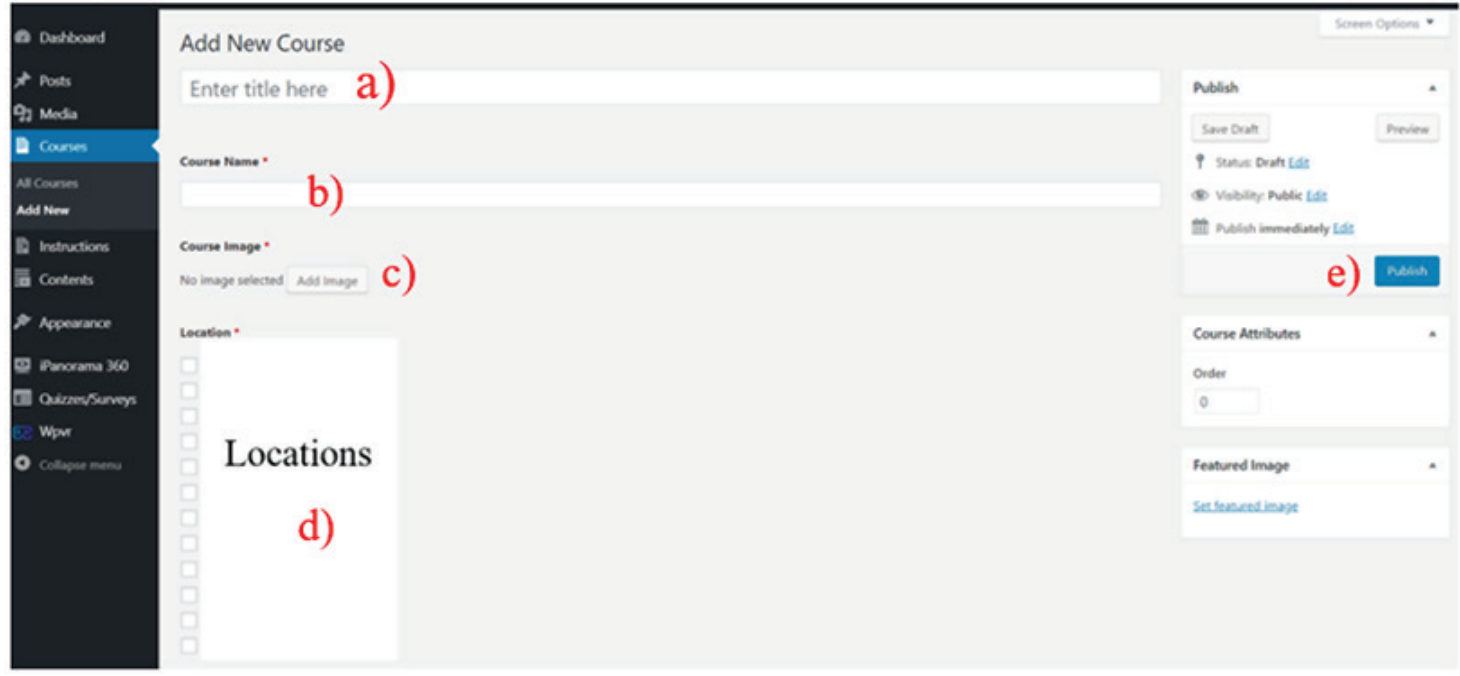

Figure 6-Adding a new course - step 1

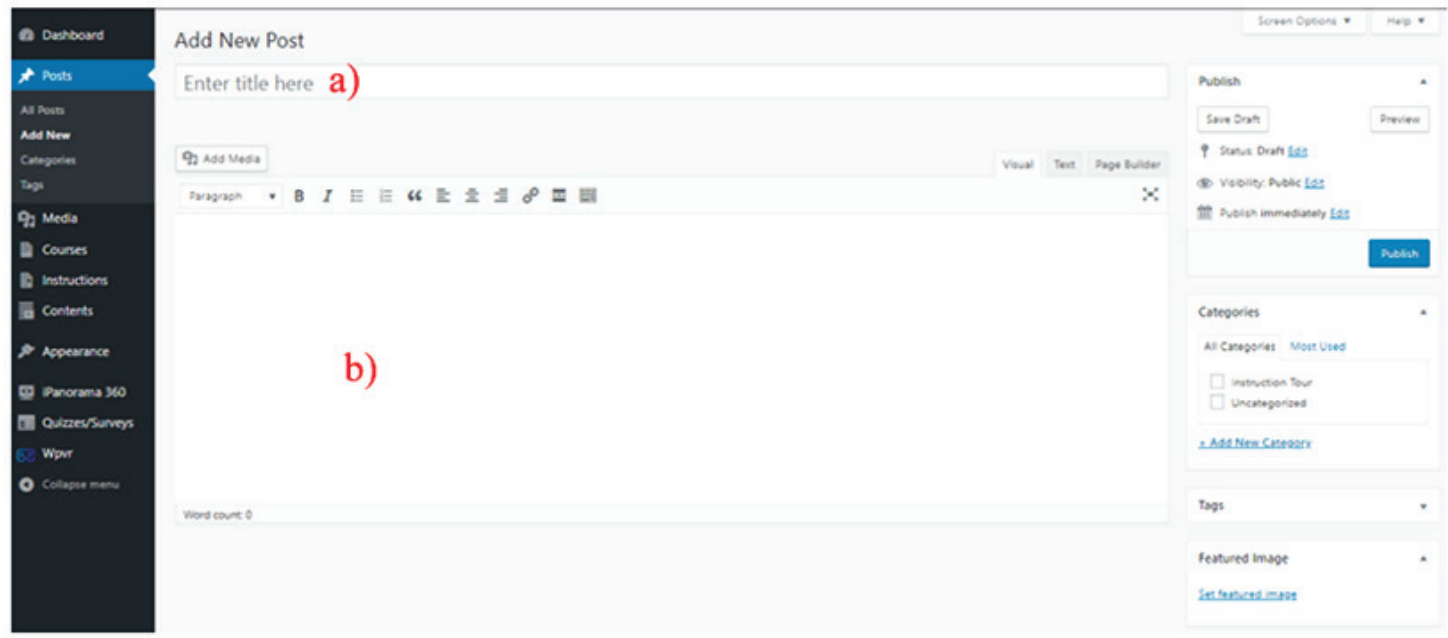

Figure 7-Creating a new post

The fourth step involves the creation of quizzes using the QSM plugin. Quiz questions will depend on training necessities and are derived from the training manuals for different machines.

The fifth step requires the use of the 'Wpvr' plugin, which contains a video player that can show 360-degree training videos. In this example application, inserted videos were 10-15 minutes long and included information on pre-shift machine inspection.

The sixth step is to connect the training course with the associated course name, relevant quizzes, and virtual tours that were created in earlier steps. Aside from these three factors, the menu option 'Instructions' is used to insert a document (training manual) into the course.

In the seventh step, the menu option is used to insert the content of the page. This includes written explanations of particular instructions and 2D images.

In the eighth step, the 'navigation menu' can be created to provide an easy, customized method of navigating the training course for future users. However, the creation of this menu is optional.

Finally, permission levels and user roles are created and assigned. In WordPress, permission levels are highly customizable based on the needs of the training software and software users. A software program can have one or more registered users. If the application is to be managed by multiple people, not every user should be authorized for full application control. As such, it is important to assign users certain roles so that every user knows their rights and permissions, as well as what changes to the application they can or cannot make. To increase the overall security of the web application, WordPress offers the ability to assign several levels of authorization to each user. This feature prevents unauthorized users from accessing the application. In addition, it does not allow the execution of tasks beyond a user's permitted duties.

The web-based training application in this study proposed the following three levels of permission for users and their roles:

$>$ Administrator role

$>$ Editor role

$>$ Trainee role.

The administrator role has the highest level of authority among all users. The administrator, who regulates the application, can monitor the complete dashboard and fully control both back-end and front-end content. Thus, an administrator 


\section{Application of a content management system for developing equipment safety training courses}

can make core changes related to coding, install new plugins, or delete existing ones. The administrator is also able to assign or change a user's roles.

The editor role is second to the administrator in terms of authority with a partial permission level. The editor can insert new content, create new training courses from the template, and modify existing courses. However, an editor cannot make essential application changes such as coding.

A trainee has the lowest permission level. Thus, a trainee is only a visitor, lacking any authority to make changes to the content of the web application, courses, plugins, or code. A trainee is also not allowed to create a new course; they can only take an existing one.

By using this approach, training courses were developed for machines that were widely used in the surface mine. The list of developed training courses is presented in Table I.

Figure 8 shows the screen where users can select one of the developed training courses. Each course is designed for training on a single machine.

Figure 9 shows that after selecting the training course, a trainee can click the button (a) to initiate training, and navigate through the selected course by using arrows or the navigation menu (d). The user can also choose another machine (b) and download a PDF version of the training manual for the selected machine (c).

\begin{tabular}{|l|c|}
\hline \multicolumn{2}{|l|}{ Table I } \\
List of developed courses \\
\hline & Machine \\
\hline 1. & Haul Truck - CAT 789 \\
2. & Haul Truck - CAT 785 \\
3. & Dozer - CAT D10 \\
4. & Dozer - CAT D11 \\
5. & Scraper - CAT 16M \\
6. & Scraper - CAT 24M \\
7. & Surface miner - Wirtgen 4200 \\
\hline
\end{tabular}

A trainee can read, listen, watch training videos, and take virtual tours related to the selected training course. Figure 10 shows an example of a virtual tour. During the virtual tour, a user can move between 360-degree images by using hotspots (a) to observe different machine parts. Hotspots can be followed by voice information about major machine parts. The system tracks what images are visited during a virtual tour. When a blue square (b) appears, it indicates that this location has been visited. Therefore, the user knows what areas of the tour are still left to be checked. A trainee can go into the non-full screen or full-screen mode (c) and use an image thumbnail slider (d) as a second option for navigating between scenes. These concepts have been applied to all the virtual tours created for the chosen

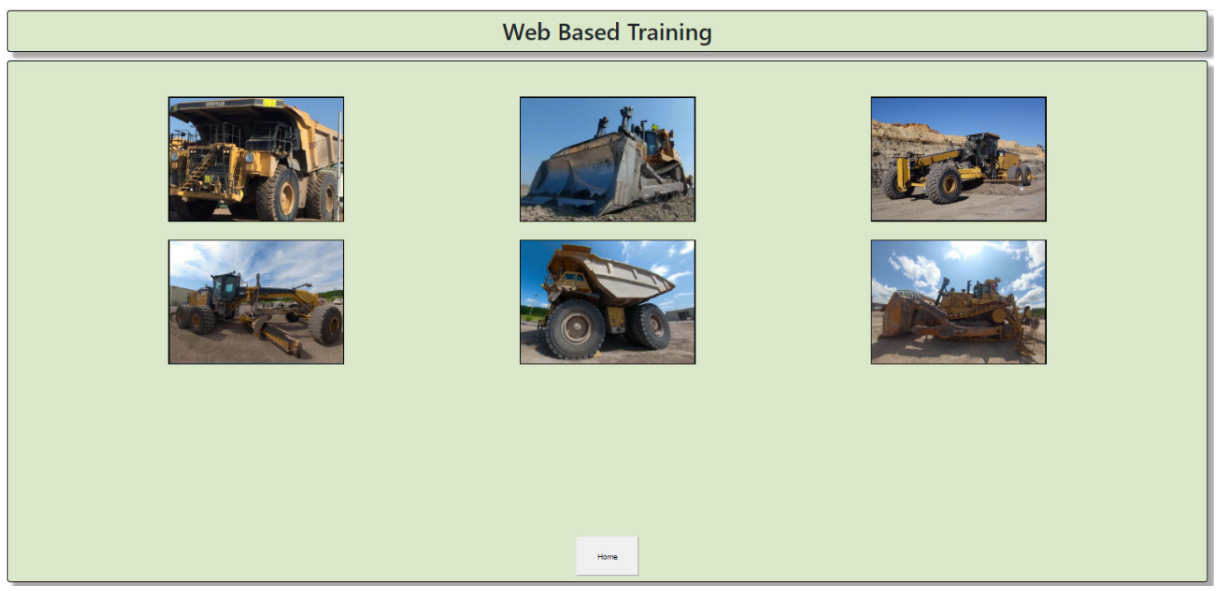

Figure 8-Course selection screen

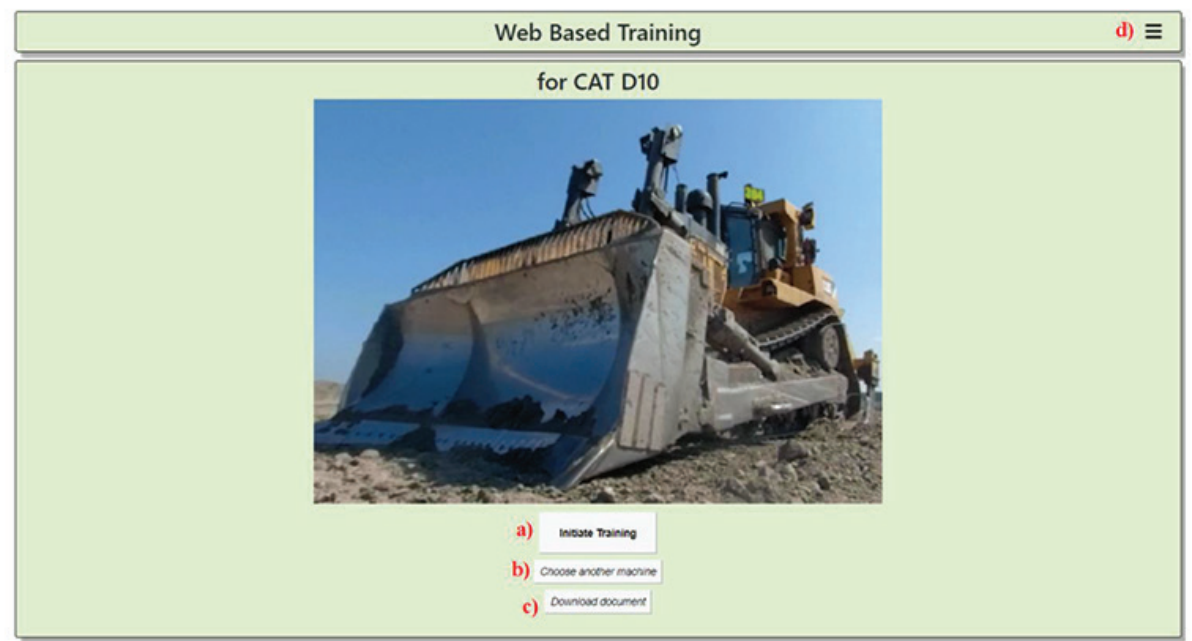

Figure 9-Training course screen 


\section{Application of a content management system for developing equipment safety training courses}

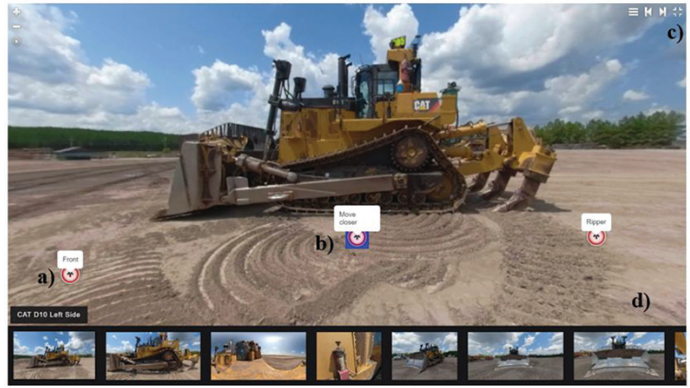

Figure 10-Virtual tour - dozer

machines. Figures 11 and 12 give examples from a 360-degree virtual tour, presenting different details that can be observed by users.

360-degree videos on the machine inspection process were inserted into the courses using the Wpvr plugin as explained earlier. Such training videos allow a person to get a closer look at a machine and learn more about the pre-shift inspection process. Video screenshots from haul truck and dozer inspections are shown in Figure 13.

It is also possible to take quizzes created with the QSM plugin. Quizzes are given at the end of training courses, assessing a trainee's knowledge about the information they received through training manuals, virtual tours, and videos. The results of the quiz are sent to an administrator by e-mail directly after a trainee submits their quiz responses; further steps should be determined by the trainer. Figure 14 shows an e-mail that includes a trainee's name, test score, time taken to finish a quiz, and the quiz questions paired with the trainee's responses.

This web application has the potential to support traditional training provided to machine operators by presenting written information, virtual tours, videos, and knowledge assessment quizzes in one place. Modifications or improvements of the training courses can also be made with ease.

The results of this research project show that it is possible to utilize CMS WordPress to develop a template and to create training courses. These in turn would help in introducing machine operators to machinery and the pre-shift machine inspection process in surface mining. Potential users of such applications can develop new training courses, or change existing courses, without needing programming skills. Users can also remove or insert materials directly into the media database.

It is beneficial to introduce machine operators to machinery and major daily procedures in the office environment before they go into the field. This will promote safety awareness. For instance, from a safety standpoint, some new operators might not have seen these machines previously, and this training approach could help familiarize them with the important parts of the machinery by using a mix of 360-degree images, videos, and training documents. The plugins and WordPress are upgraded
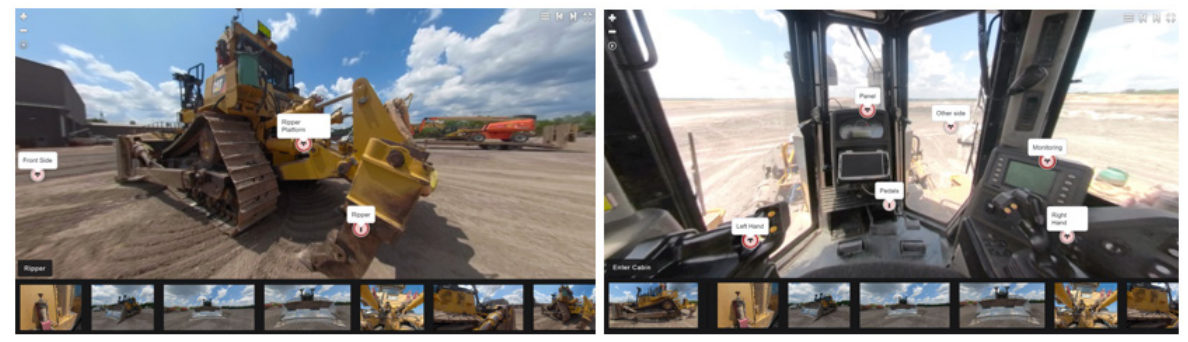

Figure 11-Dozer - side and cabin views
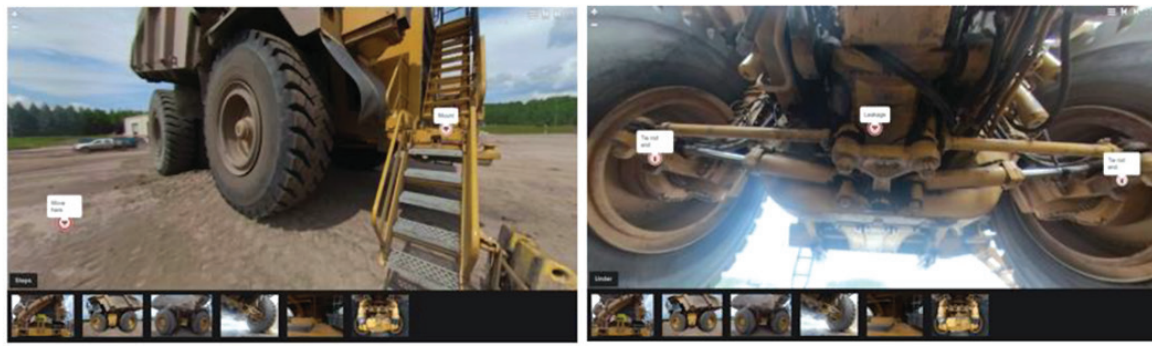

Figure 12-Haul-truck - side and underneath views
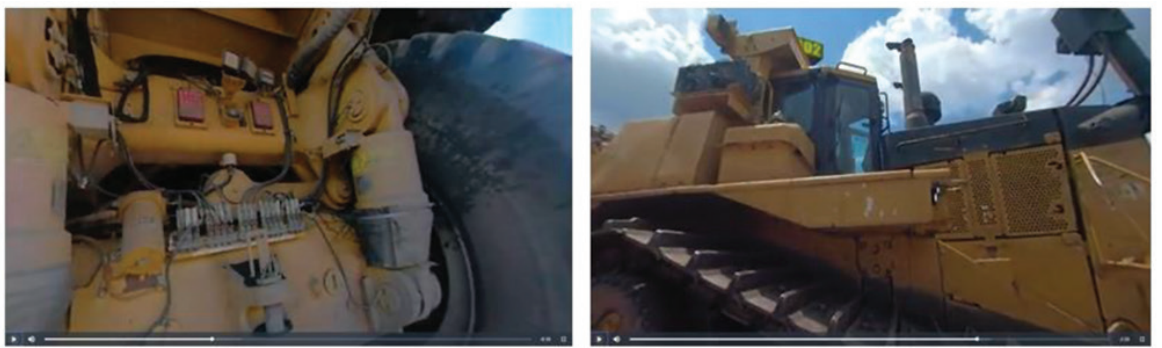

Figure 13-360-degree video - haul-truck inspection and dozer inspection 


\section{Application of a content management system for developing equipment safety training courses}

Quiz Results for CAT 789 - Final Test

Employee name: HEO Training

Employee number: 123456

Quiz Score: 818 or $100 \%$ or 8 point

The user took 65 seconds to complete quiz

Answers Provided:

\section{Question}

During your pre-shift inspection you noticed that Two Way Radio is not working. What will you do?

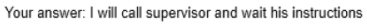

Correct answer: I vill call supervisor and wait his instruction

Question

During your shift you noticed loss of brakes on your haul truck. What will you do?

Your answer. I will pull over, stop and apply the park brake

Correct answer. I vill pull over, stop and apply the park brak

Question

You want to drive a haul truck backward. How will you signal that?

Figure 14-Quiz results sent on e-mail address

periodically to newer versions, and therefore they can be updated by a user. Ordinarily, a user would not have issues while updating the plugin. However, updates are not required, and the application is operable without newer versions of the plugins or CMS.

The developed application is not only useful for developing safety training courses related to surface mining machines. It can potentially be used for other training and educational purposes - for example, it could be used for virtual tours of a mine site, as well as in other industries.

\section{Conclusions}

The web-based application developed through this research project can support traditional safety training by introducing machine operators to the different surface mining machines they will eventually operate in the field. The application is highly flexible for creating and editing training courses. An emphasis was placed on virtual tours and 360-degree videos that could assist in learning about machines and pre-shift inspections.

The outcomes of using CMS can be summarized as follows:

> Several plugins, along with default WordPress features, are identified, explained, selected, and used to create the application and training template.

$>$ The process of developing new training courses in several steps, without prior coding skills, is explained.

- The developed application is deployed on a web server, allowing both online and offline access.

Future research and improvements to this web-based training could include:

> Further investigation and implementation of other useful plugins, which could widen the use of the developed application
> Development of custom-made plugins as a replacement for available WordPress plugins.

$>$ Discussion on further uses for the developed application.

\section{Acknowledgements}

This research was supported by a mining company in the USA.

\section{References}

Ammoyun, O. and CAreddu, N. 2014. Mine simulation for educational purposes: a case study. Computer Applications in Engineering Education, vol. 23, no. 2. pp. 286-293.

Avila, J., Sostmann, K., Breckwoldt, J., and Peters, H. 2016. Evaluation of the free, open-source software WordPress as electronic portfolio system in undergraduate medical education. BMC Medical Education, vol. 16, no. 157. pp. 1-10.

Baines, S.A. 2016. WordPress for libraries. Hafaele, C. (ed.). Journal of Access Services, vol. 13, no. 3. pp. 213-214

Brnich, M., Derick, R.L., Mallet, L., and VAught, C. 2002. Innovative alternatives to traditional classroom health and safety training. Strategies for improving miners' training. Peters, R.H. (ed.). US Department of Health and Human Services, Public Health Service, Centers for Disease Control and Prevention, National Institute for Occupational Safety and Health, DHHS (NIOSH) Publication No 2002-156, Information Circular 9463, 03 September 2002. pp. 19-25.

САвот, J. 2018. WordPress: A content management system to democratize publishing. Institute of Electrical and Electronics Engineers Software, vol. 35, no. 3. pp. 89-92.

Chamers, T., and LeE, D. 2004. Web-based training in corporations: organizational considerations. International Journal of Instructional Media, vol. 31, no. 4. pp. 345-354.

ChAn, S.C.H. and NGAI, E.W.T. 2007. A qualitative study of information technology adoption: how ten organizations adopted web-based training. Information Systems Journal, vol. 17, no. 3. pp. 289-315.

FAHY, P.J. 2004. Web-based training. The Internet Encyclopedia. Bidgoli, H. (ed.) vol. 3, pp. 661-673.

Fernandes, S. and VidyASAgar, A. 2015. Digital marketing and WordPress. Indian Journal of Science and Technology, vol. 8, no. S4. pp. 61-68.

GAo, Y., FAn, Y.X., WANG, J., LI ,X., and PEI, J.J. 2019. The mediating role of safety management practices in process safety culture in the Chinese oil industry. Journal of Loss Prevention in Process Industries, vol. 57. pp. 223-230.

GHoRecha, V. and BнAт, C. 2013. A guide for selecting content management system for web application development. International Journal of Advance Research in Computer Science and Management Studies, vol 1, no. 3. pp. 13-17.

iPanorama360. 2019. https://codecanyon.net/item/ipanorama-360-virtual-tourbuilder-for-wordpress/17028820

KRAL, S. 2002. Improved training reduces worker injuries. Mining Engineering, vol. 54, no. 10. pp. 23-26.

MAnoJ, S. and AsoKe, N. 2016. Web content management system. International Journal of Innovative Research in Advanced Engineering, vol. 3, no. 3. pp. 51-56.

NAmestovsкi. Z., TAKAcs, M., and Arsovic, B. 2012. Supporting traditional educational process with e-learning tools. Proceedings of the 10th Jubilee International Symposium on Intelligent Systems and Informatics, Subotica, Serbia, 20-22 September 2012. Institute of Electrical and Electronics Engineers. pp. 461-464.

PAtel, S.K., RATHod, V.R., and PARIKH, S. 2011. Joomla, Drupal and WordPress - a statistical comparison of open source CMS. Proceedings of the 3rd International Conference on Trends in Information Sciences \& Computing. Institute of Electrical and Electronics Engineers. pp. 182-187.

Peters, H.R., Vaught, C., and Mallet, L. 2010. A review of NIOSH and U.S. Bureau of Mines research to improve miners' health and safety training. Extracting the Science: A Century of Mining Research. Brune, J.F. (ed.). Society for Mining, Metallurgy, and Exploration, Inc., Littleton, CO. pp. 501-509.

Quiz MASTER. 2019. https://wordpress.org/plugins/quiz-master-next/

ZhANG, M. and Kecojevic, V. 2016. Intervention strategies to eliminate truck-related fatalities in surface coal mining in West Virginia. International Journal of Injury Control and Safety Promotion, vol. 23, no. 2. pp. 115-129.

WoRDPRESS. 2019. WP VR - 360 Panorama and virtual tour creator for WordPress. https://wordpress.org/plugins/wpvr/

WordPress. 2020. Features. https://wordpress.org/about/features/ 